\title{
The intervals of oscillations in the
} solutions of the Legendre differential equations

\author{
Dimitris M Christodoulou', James Graham-Eagle ${ }^{1}$ and Qutaibeh D Katatbeh ${ }^{2 *}$
}

\section{"Correspondence:}

qutaibeh@just.edu.jo

${ }^{2}$ Department of Mathematics and

Statistics, Jordan University of

Science and Technology, Irbid, 22110, Jordan

Full list of author information is available at the end of the article

\begin{abstract}
We have previously formulated a program for deducing the intervals of oscillations in the solutions of ordinary second-order linear homogeneous differential equations. In this work, we demonstrate how the oscillation-detection program can be carried out around the regular singular points $x= \pm 1$ of the Legendre differential equations. The solutions $y_{n}(x)$ of the Legendre equation are predicted to be oscillatory in $|x|<1$ for $n \geq 3$ and nonoscillatory outside of that interval for all values of $n$. In contrast, the solutions $y_{n}^{m}(x)$ of the associated Legendre equation are predicted to be oscillatory for $n \geq 3$ and $m \leq n-2$ only in smaller subintervals $|x|<x_{*}<1$, the sizes of which are determined by $n$ and $m$. Numerical integrations confirm that such subintervals are distinctly smaller than $(-1,+1)$.
\end{abstract}

MSC: 34A25; 34A30; 42C05

Keywords: oscillations; second-order linear differential equations; analytical theory; transformations; Legendre differential equations

\section{Introduction}

It is well known (e.g., [1], Section 10.2; [2], Section XI.1) that all ordinary second-order linear homogeneous differential equations can be written in the general form

$$
y^{\prime \prime}+b(x) y^{\prime}+c(x) y=0
$$

and that this form can be transformed to the canonical form

$$
u^{\prime \prime}+q(x) u=0
$$

where the primes denote derivatives with respect to the independent variable $x, q=$ $-\left(b^{2}+2 b^{\prime}-4 c\right) / 4$, and $y(x)=u(x) \exp \left(-\frac{1}{2} \int b(x) d x\right)$. The canonical form (2) condenses all the information regarding the coefficients of equation (1) into $q(x)$ and 'oscillation theory' focuses on this coefficient in order to derive the oscillatory properties of the solutions of equation (1) (see the reviews in [3, 4] and references therein). In a previous paper ([5], hereafter CGK), we also focused on the $q(x)$ of equation (2). We formulated a new definition of oscillation as the appearance of successive critical points of the same kind (maxima, or

(c) 2016 Christodoulou et al. This article is distributed under the terms of the Creative Commons Attribution 4.0 International License (http://creativecommons.org/licenses/by/4.0/), which permits unrestricted use, distribution, and reproduction in any medium, provided you give appropriate credit to the original author(s) and the source, provide a link to the Creative Commons license, and indicate if changes were made. 
minima, or inflection points) in the graph of a solution. Then we described a program for deducing the precise intervals of oscillations in the solutions of the general form (1), and we presented a variety of examples in which this procedure was successful in predicting oscillatory behavior by examining the coefficient $q(x)$ alone.

Some of the differential equations that were investigated by CGK were transformed to the harmonic-oscillator form with constant coefficients in the first step of the program, and their oscillatory properties were then derived easily from the discriminant of their characteristic quadratic equations. The rest of the equations were transformed to a form with constant damping (a constant coefficient of the first-derivative term), a quantity that clearly opposes any oscillatory tendencies in the solutions. In this second step of the program, a generalized Euler transformation of the independent variable $x$ was used:

$$
x=c_{1}+c_{2} \exp (k t)
$$

where $c_{1}, c_{2}$, and $k$ are arbitrary constants, and a criterion for the intervals of oscillations in the solutions was established:

$$
q(x)>\frac{1}{4\left(x-c_{1}\right)^{2}}
$$

Only the constant $c_{1}$ appears in the criterion and corresponds to a 'horizontal shift' of the independent variable $x$ in equation (3).

The equations studied by CGK in the second step of the program contained singularities at $x=0$ or no singularities at all, in which cases $c_{1}$ was set to zero in equations (3) and (4). In the present work, we extend our investigation to the study of oscillatory properties of the Legendre and associated Legendre equations $[6,7]$ that have regular singular points at $x= \pm 1$ (that is, away from $x=0$ ). In these cases, a horizontal shift $c_{1} \neq 0$ proves to be quite useful, since it can be chosen to circumvent one or the other singularity in the neighborhood of which the intervals of oscillations in the solutions are being sought. The other two constants in equation (3) are chosen in ways that allow for the investigation of specific intervals in $x$ (in particular, $0 \leq x<1$ and $1<x<\infty$ ). We use the above equations in Section 2 and Section 3 below, where we analyze the Legendre equation and the associated Legendre equation, respectively. Finally, in Section 4, we summarize our results.

\section{Legendre equation}

The Legendre equation $[6,7]$

$$
y^{\prime \prime}-\frac{2 x}{1-x^{2}} y^{\prime}+\frac{n(n+1)}{1-x^{2}} y=0,
$$

where $n$ is a constant, is first transformed to the canonical form (2) in which the coefficient $q(x)$ is

$$
q(x)=\frac{n(n+1)\left(1-x^{2}\right)+1}{\left(1-x^{2}\right)^{2}},
$$

and $u(x)=y(x) \sqrt{\left|1-x^{2}\right|}$. The coefficient $q(x)$ is an even function of $x$, so we can investigate the interval $x \geq 0$ and the results will be applicable for $x<0$ as well. 
We choose $c_{1}=1$ and $k=1$ in equation (3), in which case the transformation becomes

$$
x=1+c_{2} \exp (t)
$$

and the criterion (4) for oscillatory solutions becomes

$$
q(x)>\frac{1}{4(x-1)^{2}}
$$

By setting $c_{2}$ to -1 or +1 in equation (7), we can search for oscillatory solutions in intervals on either side of $x=1$. Specifically, for $c_{2}=-1$ :

$$
t \in(-\infty, 0] \quad \Longrightarrow \quad x \in[0,+1)
$$

and for $c_{2}=+1$ :

$$
t \in(-\infty,+\infty) \Longrightarrow x \in(+1,+\infty)
$$

In both cases, equation (8) is the applicable criterion for oscillatory solutions, so these two intervals in $x$ can be investigated simultaneously. The pole at $x=+1$ is eliminated from the analysis when equations (6) and (8) are combined, in which case we find that the interval over which the solutions may oscillate is given by the solution of the algebraic inequality

$$
N x^{2}+2 x-(N+2)<0 \quad \Longrightarrow \quad x \in(-1-2 / N,+1)
$$

where

$$
N \equiv(2 n+1)^{2}
$$

The endpoint $x_{-}=-1-2 / N$ lies on the negative side of the $x$-axis, therefore oscillatory solutions are predicted in the entire interval $x \in[0,+1)$. Furthermore, all solutions in $x \in$ $(+1,+\infty)$ are predicted to be nonoscillatory.

Using the even symmetry of the canonical form (2) with $q(x)$ given by equation (6), we conclude that the solutions of the Legendre equation can oscillate only in the interval

$$
|x|<1
$$

On the other hand, we know from the results obtained by CGK that low-order polynomial solutions will be nonoscillatory and that the lowest-order solutions that may exhibit at least two critical points of the same kind (maxima, or minima, or inflection points) for certain choices of boundary conditions have $n=3$. The borderline case with $n=3$ is discussed in the Nodal analysis below.

Numerical results. Figures 1 and 2 show two low-order numerical solutions of equation (5) in $(-1,+1)$. In Figure $1, n=3$ and the boundary conditions are $y(0)=1$ and $y^{\prime}(0)=0$. In Figure 2, $n=4$ and the boundary conditions are $y(0)=0$ and $y^{\prime}(0)=1$. In both cases, the solutions are oscillatory according to our definition since they exhibit two extrema of the same kind. Additional numerical integrations confirm that, for larger values of $n$, the solutions exhibit increasingly more oscillations in $(-1,+1)$ while the solutions outside of that interval are nonoscillatory for all values of $n$. 


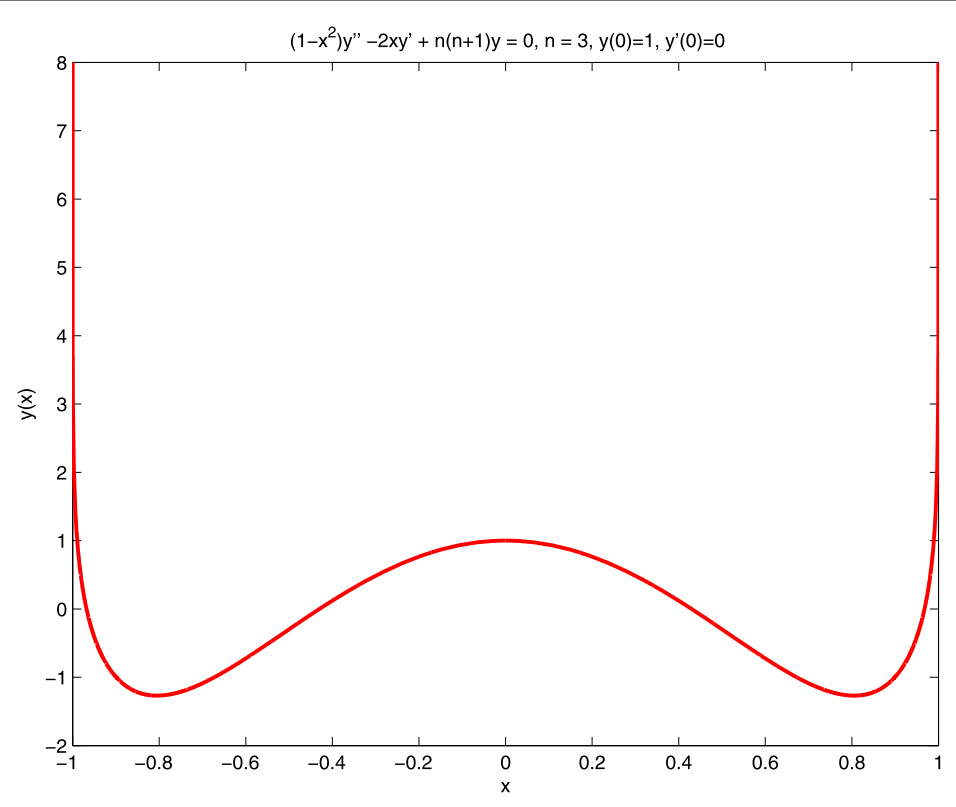

Figure 1 The numerical solution of equation (5) with $n=3$ and boundary conditions $y(0)=1$ and $y^{\prime}(0)=0$.

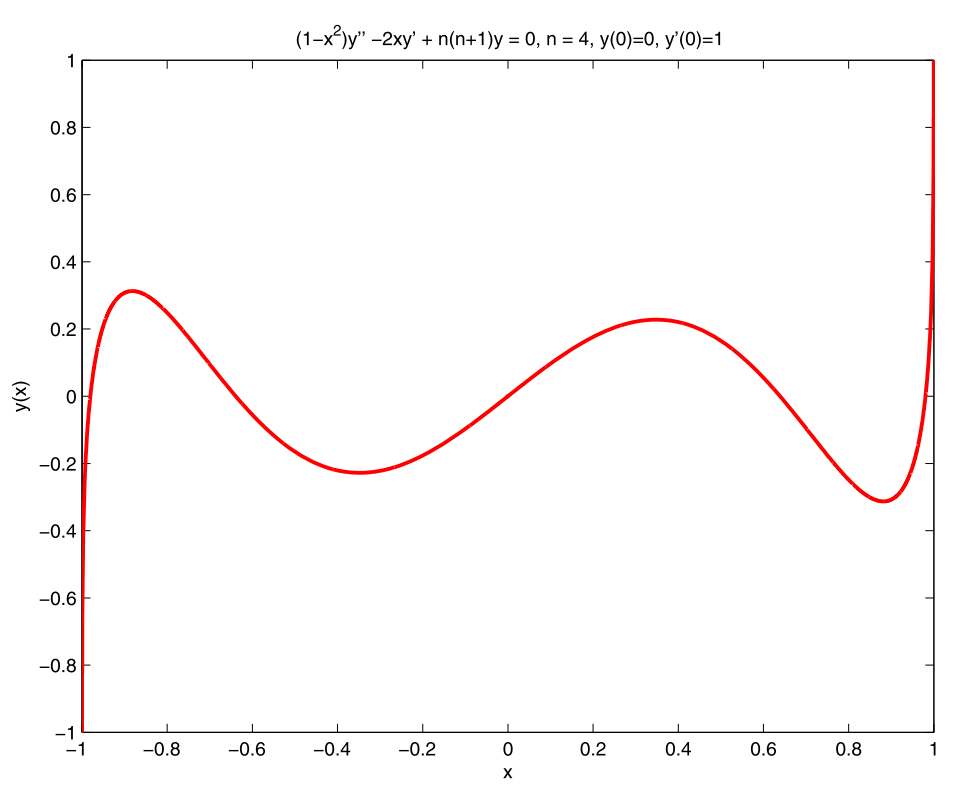

Figure 2 The numerical solution of equation (5) with $n=4$ and boundary conditions $y(0)=0$ and $y^{\prime}(0)=1$.

Nodal analysis. The above results can be better understood by counting the number of nodes $\mathcal{N}(n)$ in $x \in[-1,+1]$ for the two types of boundary conditions used. For $y(0)=1$, the solutions are even and the number of nodes is

$$
\mathcal{N}(n)= \begin{cases}n, & \text { if } n \text { is even, } \\ n+1, & \text { if } n \text { is odd }\end{cases}
$$


while for $y(0)=0$, the solutions are odd and then the number of nodes is

$$
\mathcal{N}(n)= \begin{cases}n+1, & \text { if } n \text { is even, } \\ n, & \text { if } n \text { is odd }\end{cases}
$$

where the node at $x=0$ has been counted for the odd solutions of equation (13). Nodes at $x= \pm 1$ do not occur for any $n$, not even in solutions that remain finite at these endpoints.

The node counts clarify why the lowest-order oscillatory solutions occur for $n=3$ : the even solution has $\mathcal{N}(3)=4$ and develops two minima, so it is oscillatory. On the other hand, the odd solution has $\mathcal{N}(3)=3$ and two extrema of the same kind do not occur, so it is nonoscillatory. For $n \geq 4$, both types of solutions have enough nodes to guarantee oscillatory behavior, and the number of nodes and oscillations increases with increasing $n$.

\section{Associated Legendre equation}

The associated Legendre equation $[6,7]$

$$
y^{\prime \prime}-\frac{2 x}{1-x^{2}} y^{\prime}+\left[\frac{n(n+1)}{1-x^{2}}-\frac{m^{2}}{\left(1-x^{2}\right)^{2}}\right] y=0
$$

where $n$ and $m$ are constants, is first transformed to the canonical form (2) in which the coefficient $q(x)$ is

$$
q(x)=\frac{n(n+1)\left(1-x^{2}\right)+\left(1-m^{2}\right)}{\left(1-x^{2}\right)^{2}}
$$

and $u(x)=y(x) \sqrt{\left|1-x^{2}\right|}$. The coefficient $q(x)$ is an even function of $x$, so we can investigate the interval $x \geq 0$ and the results will be applicable for $x<0$ as well.

We choose again $c_{1}=1$ and $k=1$ in equation (3), in which case the transformation (7) is applicable and the criterion for oscillatory solutions in $x \in[0,+\infty)$ is given by inequality (8). Substituting equation (15) into equation (8), we find that the interval over which the solutions may oscillate is given by the solution of the algebraic inequality

$$
N x^{2}+2 x-(N+2 M)<0 \quad \Longrightarrow \quad x \in\left(x_{-}, x_{+}\right)
$$

where

$$
x_{ \pm}=\frac{1}{N}\left(-1 \pm \sqrt{N^{2}+2 M N+1}\right),
$$

$N$ is given by equation (10), and

$$
M \equiv 1-2 m^{2} .
$$

The endpoint $x_{-}$lies on the negative side of the $x$-axis, therefore oscillatory solutions are predicted in the interval $x \in\left[0, x_{+}\right)$. Furthermore, all solutions in $x \in\left(x_{+},+\infty\right)$ are predicted to be nonoscillatory.

Using the even symmetry of the canonical form (2) with $q(x)$ given by equation (15), we conclude that the solutions of the associated Legendre equation can oscillate only in the 
interval $|x|<x_{*}$, where from equation (17):

$$
x_{*} \equiv x_{+}=\frac{1}{N}\left(-1+\sqrt{N^{2}+2 M N+1}\right) .
$$

It is easy to show that $x_{*}<1$ for all values of $m \neq 0$. For $m=0$, then $M=1$ and equation (19) reduces to $x_{*}=1$, as was also found in Section 2 above for the Legendre equation. Therefore, in all cases of interest (in which $m \neq 0$ and $|m| \leq|n|)$, the interval $\left(-x_{*},+x_{*}\right)$ is a distinct subinterval of $(-1,+1)$ and the oscillations of the solutions of the associated Legendre equation will have to cease before the solution curves approach the endpoints $x= \pm 1$. Indeed, this behavior is observed in the numerical solutions of various cases with $m \geq 1$.

Numerical results. Figures 3 and 4 show two numerical solutions of equation (14) that oscillate in a distinct subinterval of $(-1,+1)$. In Figure $3, n=5, m=2$, and the boundary conditions are $y(0)=1$ and $y^{\prime}(0)=0$. In Figure $4, n=12, m=8$, and the boundary conditions are $y(0)=0$ and $y^{\prime}(0)=1$. Additional numerical integrations show that, for larger values of $n$ and a fixed value of $m$, the solutions exhibit increasingly more oscillations in $\left(-x_{*},+x_{*}\right)$ while the solutions outside of that interval are nonoscillatory for all values of $n$. Furthermore, for a fixed $n$ and increasing $m$, the oscillation interval $\left(-x_{*},+x_{*}\right)$ shrinks and the solutions exhibit progressively fewer nodes. Finally, for $m \geq n-2$, some solutions do appear that are nonoscillatory over the entire interval $(-1,+1)$. In the borderline case with $m=n-2$, such nonoscillatory solutions depend critically on the adopted boundary conditions (see Figures 5 and 6), while for $m>n-2$, all solutions are nonoscillatory (see the Nodal analysis below). Two examples of the borderline case with $m=n-2=10$ are shown in Figures 5 and 6 . The odd solution in Figure 5 is nonoscillatory while the even solution in Figure 6 exhibits two minima. Even in cases such as that of Figure 5, however,

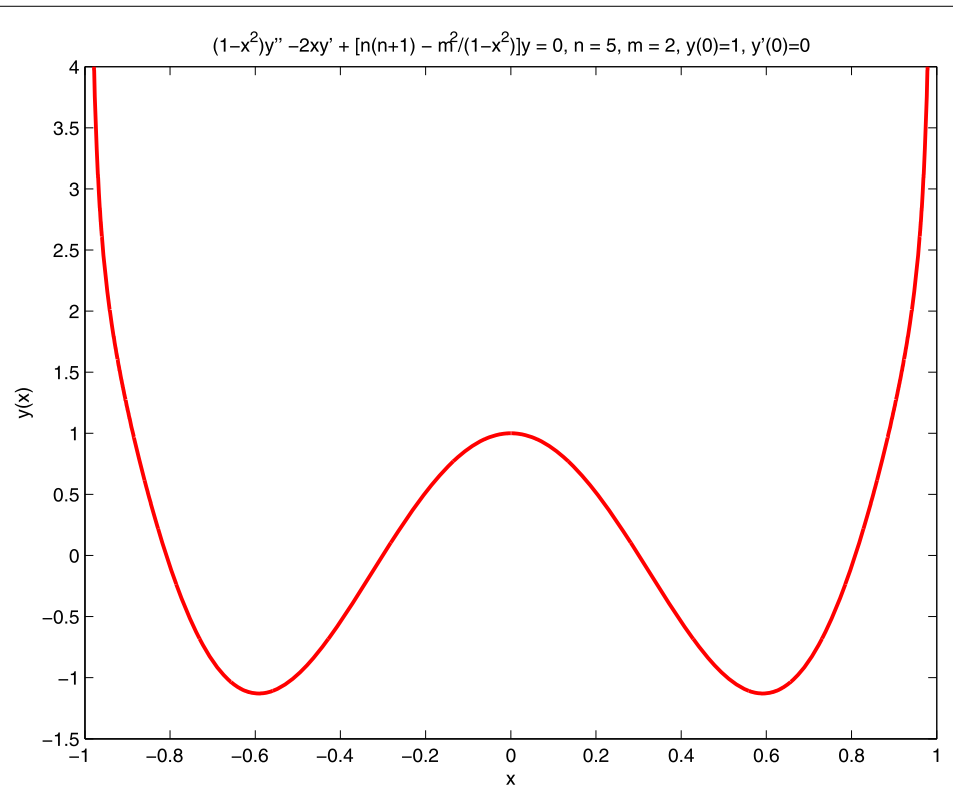

Figure 3 The numerical solution of equation (14) with $n=5, m=2$, and boundary conditions $y(0)=1$ and $\boldsymbol{y}^{\prime}(\mathbf{0})=\mathbf{0}$. The oscillations terminate at $x_{*} \approx \pm 0.93$, as predicted by equation (19). 


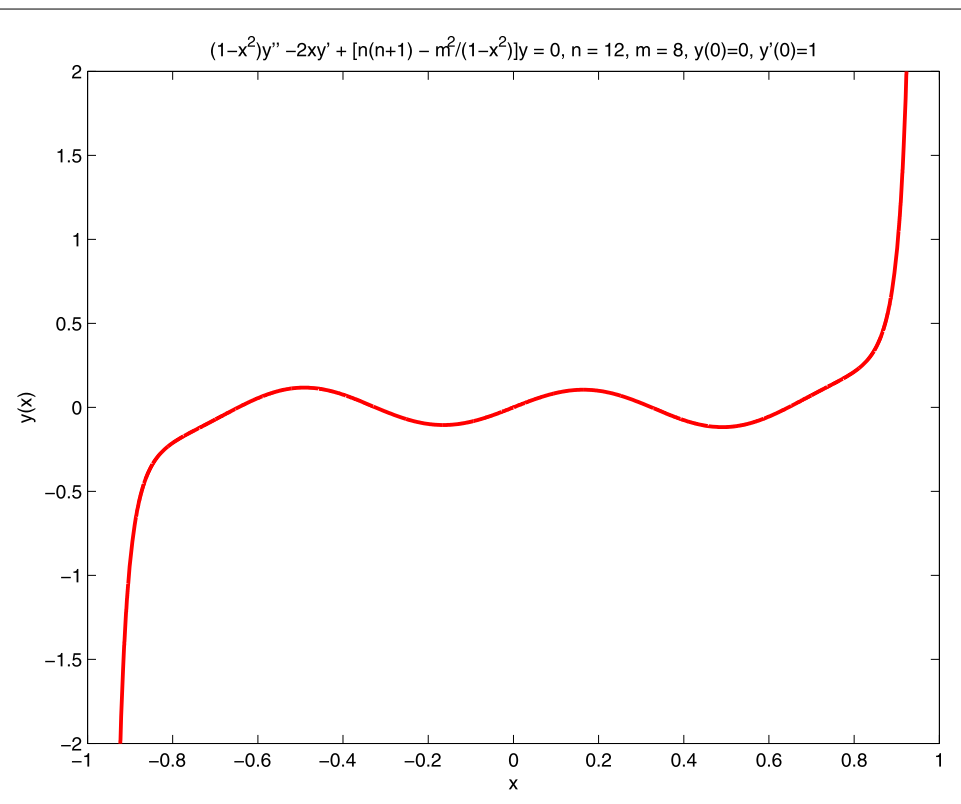

Figure 4 The numerical solution of equation (14) with $n=12, m=8$, and boundary conditions $y(0)=0$ and $\boldsymbol{y}^{\prime}(\mathbf{0})=\mathbf{1}$. The oscillations terminate at $x_{*} \approx \pm 0.77$, as predicted by equation (19).

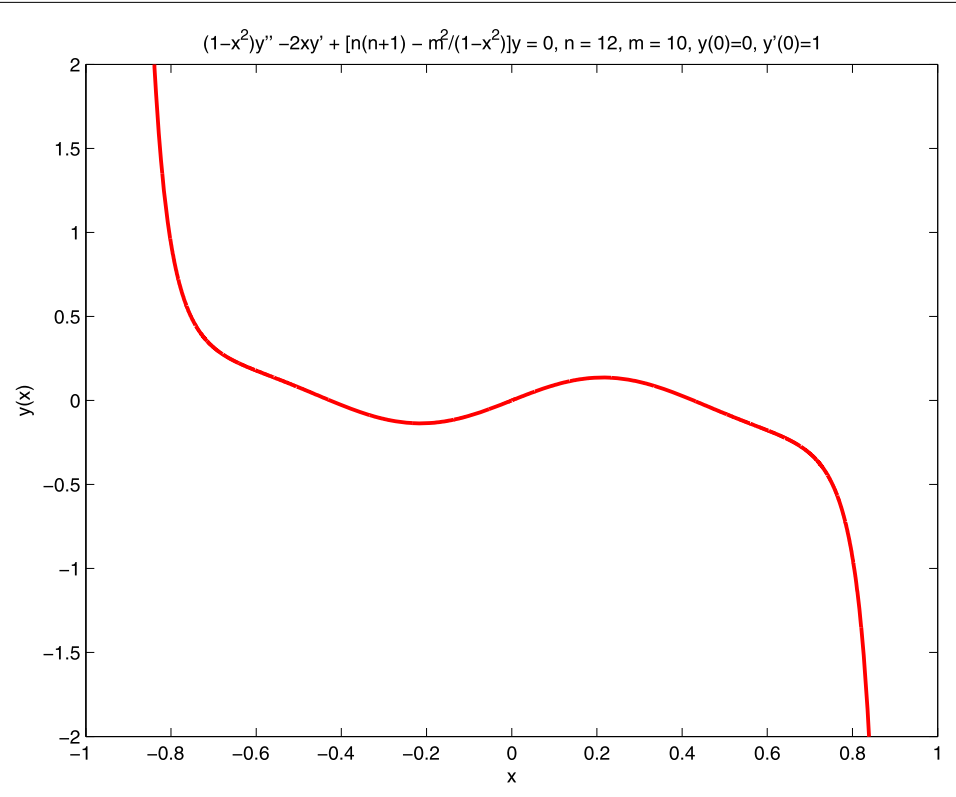

Figure 5 The numerical solution of equation (14) with $n=12, m=n-2=10$, and boundary conditions $y(0)=0$ and $y^{\prime}(0)=1$. The solution is nonoscillatory as two extrema of the same kind do not appear in the interval $(-0.60,+0.60)$ that is determined from equation (19).

the nonoscillatory solutions are distinctly nonmonotonic within the intervals specified by equation (19).

Nodal analysis. The above results can be better understood by counting the number of nodes $\mathcal{N}(n, m)$ in $x \in[-1,+1]$ for the two types of boundary conditions used. For $y(0)=1$, 


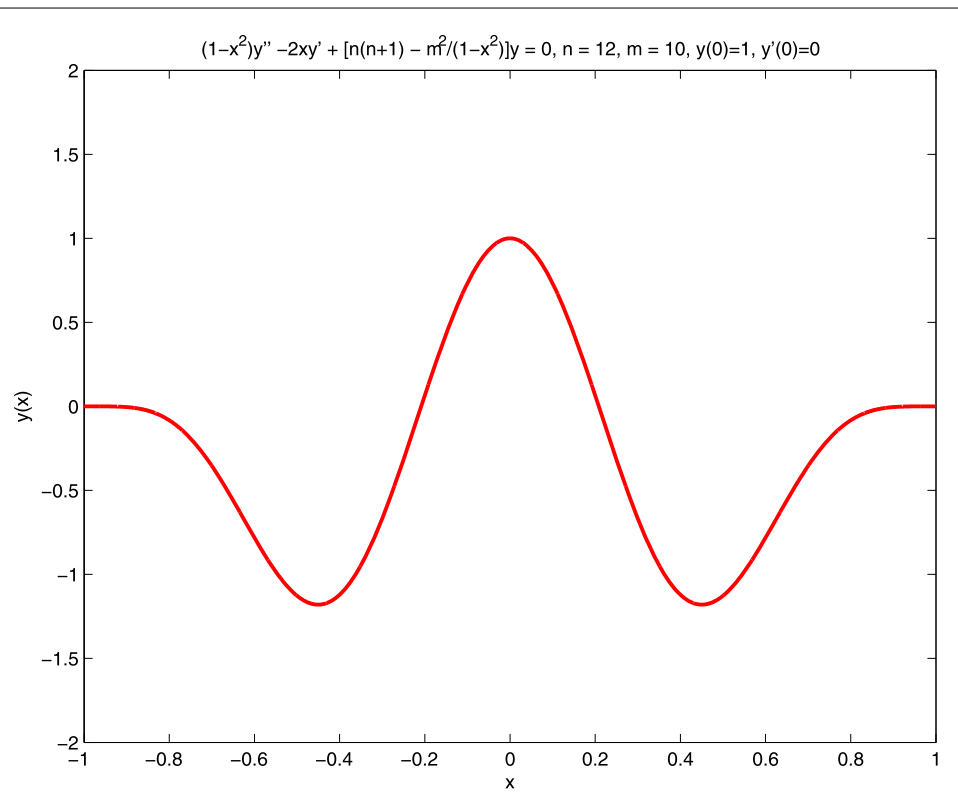

Figure 6 As in Figure 5 with $m=n-2=10$, but for different boundary conditions $y(0)=1$ and

$y^{\prime}(\mathbf{0})=\mathbf{0}$. The solution is oscillatory in the interval $(-0.60,+0.60)$, which is determined from equation (19).

the solutions are even and for $1 \leq m \leq n$, the number of nodes is

$$
\mathcal{N}(n, m)= \begin{cases}n-m+2, & \text { if } n-m \text { is even } \\ n-m+1, & \text { if } n-m \text { is odd }\end{cases}
$$

while for $y(0)=0$, the solutions are odd and for $1 \leq m \leq n$, the number of nodes then is

$$
\mathcal{N}(n, m)= \begin{cases}n-m+1, & \text { if } n-m \text { is even, } \\ n-m+2, & \text { if } n-m \text { is odd }\end{cases}
$$

where the node at $x=0$ has been counted for the odd solutions of equation (21). The term +2 in both cases represents two nodes that occur at $x= \pm 1$. For $m \neq 0$, such nodes always occur for even $n-m$ in even solutions (equation (20)) and for odd $n-m$ in odd solutions (equation (21)).

The node counts clarify the borderline case with $m=n-2$. In this case, $n-m=2$ (even) and the even solutions all have 4 nodes (equation (20)), just enough for two extrema of the same kind to develop; so they are oscillatory. In contrast, the odd solutions have 3 nodes (equation (21)) and they are all nonoscillatory. Finally, for $m>n-2$, the solutions have a very small number of nodes $(1 \leq \mathcal{N}(n, m) \leq 3)$ and then oscillations cannot occur.

Alternative analysis of oscillations. The results shown in Figures 3-6 have been verified by an independent analysis in $x \in(-1,+1)$ as follows: For $|x|<1$, the substitution

$$
x=\sin (t)
$$

transforms the associated Legendre equation (14) to the form

$$
\ddot{y}-\tan (t) \dot{y}+\left[n(n+1)-m^{2} \sec ^{2}(t)\right] y=0 \quad(|t|<\pi / 2),
$$


where dots denote derivatives of $y(t)$ with respect to $t$. The canonical form (2) of this equation has $u(t)=y(t) \sqrt{\cos (t)}$ and

$$
q(t)=\frac{1}{4}\left[N+(2 M-1) \sec ^{2}(t)\right],
$$

where $N$ and $M$ are given by equations (10) and (18), respectively. Since these equations are strictly applicable in $|t|<\pi / 2$, then the criterion (4) for oscillatory solutions can be used with $c_{1}=0$, in which case it reads

$$
q(t)>\frac{1}{4 t^{2}}
$$

Substituting equation (24) into equation (25), we find that oscillatory solutions can occur in a subinterval of $|t|<t_{*}<\pi / 2$ in which

$$
N+(2 M-1) \sec ^{2}(t)-\frac{1}{t^{2}}>0,
$$

or, using equation (22), in a subinterval of $|x|<x_{*}<1$ in which

$$
N+\frac{2 M-1}{1-x^{2}}-\frac{1}{\left[\sin ^{-1}(x)\right]^{2}}>0 .
$$

We have solved this inequality numerically for various values of $N$ and $M$, and the resulting values of $x_{*}$ for all cases, including those depicted in Figures 3-6, are virtually identical to those calculated analytically from equation (19). The agreement between results in the above two analyses justifies the use of a horizontal shift $\left(c_{1}=1\right)$ in the former analysis. Using horizontal shifts to remove singularities simplifies the calculations and leads to quadratic inequalities that can be solved analytically.

\section{Summary}

We have presented an analysis of the oscillatory properties of the solutions of the Legendre and associated Legendre differential equations $[6,7]$ that show two regular singular points in their coefficients away from $x=0$ (at $x= \pm 1$ ). The analysis makes use of a program that was described in [5] by CGK for investigating the $x$-intervals in which the solutions have an oscillatory character. According to this procedure, an attempt must first be made to transform a given equation to a form with constant coefficients. Unlike in the case of the Chebyshev equation that also has two poles at $x= \pm 1$ but can be recast into a form with constant coefficients (CGK), the Legendre equations cannot be simplified in this manner and their analysis must proceed to the second step of the program, where a transformation of $x$ is applied in order to cast these equations into forms with constant damping (i.e., a constant coefficient of the first-derivative term). As was described in Sections 2 and 3 above, such a transformation is capable of eliminating from the analysis one or the other singularity, but not both of them at the same time. This leaves one pole in the coefficient of the canonical form of the final equation (equation (6) and equation (15)) and its presence complicates the search for oscillatory solutions. Because the coefficients (6) and (15) of the canonical form are even functions of $x$, it turns out that we can investigate only the interval $x \geq 0$ (which can be made free from the singularity at $x=+1$ ), and then use symmetry to derive the corresponding results for $x<0$. 
The results of our analysis are as follows:

(a) The solutions $y_{n}(x)$ of the Legendre equation are predicted to be oscillatory in $|x|<1$ for $n \geq 3$ (according to the definition of oscillation given by CGK and adopted here as well) and nonoscillatory outside of that interval for all values of $n$. Numerical integrations of many cases with a variety of boundary conditions confirm these predictions. Two examples of oscillatory solutions in $(-1,+1)$ are shown in Figures 1 and 2 .

(b) The solutions $y_{n}^{m}(x)$ of the associated Legendre equation are predicted to be oscillatory for $n \geq 3$ only and $m \leq n-2$ in smaller subintervals of $|x|<x_{*}<1$, where $x_{*}$ is given by equation (19). (Note, however, that in the borderline case with $m=n-2$, only the even solutions are oscillatory.) Numerical integrations, as well as a separate analysis of the alternative form (23) of the associated Legendre equation, confirm that such subintervals are predicted correctly and that they are distinctly smaller than the interval $(-1,+1)$ of the $m=0$ case. Three examples of oscillatory solutions are shown in Figures 3, 4, and 6 and an example of a nonoscillatory solution is shown in Figure 5.

In the example of Figure 5 with $m=n-2=10$, two extrema of the same kind do not occur in the predicted interval $|x|<0.60$ because of the relatively large value of $m$. In general, in the borderline case with $m=n-2$, some solutions are oscillatory and other solutions are not, depending on the adopted boundary conditions (as in Figures 6 and 5, respectively); but for $m>n-2$, all solutions are nonoscillatory because they exhibit just a small number of nodes (1 to 3 ). Even these types of solutions are, however, characteristically nonmonotonic as they attempt to oscillate, but the small number of nodes does not permit a full 'cycle' to develop in the intervals $|x|<x_{*}$ with $x_{*}$ specified by equation (19).

Competing interests

The authors declare that they have no competing interests.

Authors' contributions

All authors drafted the manuscript, and they read and approved the submitted version.

\title{
Author details
}

'Department of Mathematical Sciences, University of Massachusetts Lowell, Lowell, MA 01854, USA. ${ }^{2}$ Department of Mathematics and Statistics, Jordan University of Science and Technology, Irbid, 22110, Jordan.

\author{
Acknowledgements \\ During this research project, DMC and JG-E were supported by the University of Massachusetts Lowell while QDK was on \\ a sabbatical visit and was fully supported by the Jordan University of Science and Technology. \\ Received: 25 October 2015 Accepted: 31 January 2016 Published online: 17 February 2016 \\ References \\ 1. Whittaker, ET, Watson, GN: A Course of Modern Analysis, 3rd edn. Cambridge University Press, Cambridge (1920) \\ 2. Hartman, P: Ordinary Differential Equations. Wiley, New York (1964) \\ 3. Agarwal, RP, Grace, SR, O'Regan, D: Oscillation Theory for Second Order Linear, Half-Linear, Superlinear and Sublinear \\ Dynamic Equations. Kluwer Academic, Dordrecht (2002) \\ 4. Wong, JSW: On second-order nonlinear oscillation. Funkc. Ekvacioj 11, 207-234 (1968) \\ 5. Christodoulou, DM, Graham-Eagle, J, Katatbeh, QD: A program for predicting the intervals of oscillations in the \\ solutions of ordinary second-order linear homogeneous differential equations. Adv. Differ. Equ. (2016). \\ doi:10.1186/s13662-016-0774-x (CGK) \\ 6. Abramowitz, M, Stegun, IA (eds.): Handbook of Mathematical Functions with Formulas, Graphs, and Mathematical \\ Tables. Dover, New York (1972) \\ 7. Bell, WW: Special Functions for Scientists and Engineers. Dover, New York (2004)
}

